\title{
М. Г. Алишаев
}

\section{Применение преобразования Лапласа и его обращения для задач извлечения геотермального тепла}

\begin{abstract}
Отмечаются трудности численной реализации обращения изображения по Лапласу и указываются источники их возникновения. В качестве первого примера приводится изменение граничного условия в автомодельной задаче извлечения сухого тепла пород. Показывается, что если температура на границе постепенно нарастает с начального значения по экспоненциальному закону к тому же постоянному значению, то на отрицательной действительной оси параметра преобразования появляется полюс, интегралы по отрицательной действительной оси становятся несобственными и растут погрешности вычислений. Предлагается решение новой задачи о росте температуры термальной воды, фонтанирующей во времени с постоянной скоростью. Задача контактная, включающая как температуру горной породы с геотермическим градиентом, так и температуру жидкости в трещине. Она моделируется по схеме Ловерье, решается в нестационарной постановке с применением преобразования Лапласа, приводится к универсальному виду в безразмерных переменных. Отмечаются особенности, вносимые геотермальным градиентом и температурным фронтом воды в процедуру обращения изображения по Лапласу.

Библиография: 7 названий.
\end{abstract}

Difficulties of numerical implementation of processing Laplace image are marked and the sources of their origin are pointed out. As a first example the change of the boundary conditions in the similarity problem of extracting dry heat from rocks is given. It is shown that if the temperature on bound gradually increases from initial value to the same constant value exponentially, then the pole appears on negative real axis of the transform parameter, and the integrals on negative axis become improper and the calculation errors increase. The solution of new problem of growth of temperature of thermal water, which gushes at constant velocity in time, is proposed. This is contact problem, which includes both the temperature of rock with the geothermal gradient and the temperature of water in a fracture. It is modeled by Loverier scheme, solved in non-stationary formulation with using Laplas transform and reduced to universal form in dimensionless variables. The features, which are generated with geothermal gradient and water temperature front, are considered.

Bibliography: 7 items.

Ключевые слова: горная порода, трещина, жидкость, температура, поток.

Keywords: rock, fracture, liquid, temperature, flow. 


\section{Введение}

В нефтепромысловой механике для решения ряда линейных краевых задач тепло- и массопереноса широко применяется преобразование Лапласа и его обращение $[1,2]$. Задача отыскания точного выражения для изображения по Лапласу является более простой и менее трудоёмкой, и для большого класса функций удаётся найти точное представление изображения [1]. Есть готовые таблицы обращений для изображений [3], есть и формальное представление в квадратурах по контуру комплексной плоскости для любого изображения, т.е. для оригинала, однако численное получение результата представляет иногда значительные трудности. Трудность состоит в том, что интегрирование по контуру в комплексной плоскости оказывается недостаточно привычным делом при современном математическом образовании инженеров. Численное обращение преобразования Лапласа остаётся одной из актуальных проблем вычислительной математики, несмотря на то, что многие математические программные пакеты (Mathcad, MATLAB и др.) содержат операторы обращения изображения по Лапласу. Зачастую успех, сосчитает или откажет пакет Mathcad обращение данного изображения, зависит от выбора контура интегрирования.

Сначала мы приведём простой пример извлечения тепла горных пород, который демонстрирует метод Лапласа и появление вычислительных трудностей использования формулы обращения Меллина при изменении граничных условий. Затем дадим постановку и решение эталонной нестационарной задачи определения температуры жидкости при её добыче по вертикальной трещине.

\section{1. Пример использования преобразования Лапласа}

Одномерную задачу извлечения теплового потока из однородно нагретого полупространства горных пород описывается уравнением теплопроводности (см. [4, 5])

$$
\frac{\partial T}{\partial t}=a \frac{\partial^{2} T}{\partial x^{2}}, x>0, t>0, T(x, 0)=0, T(\infty, t)=0
$$

с вариантами граничного условия на границе $x=0$

$$
\text { 1) } T(0, t)=-\Delta T_{0}, \quad \text { 2) } T(0, t)=-\Delta T_{0}\left(1-e^{-\alpha t}\right) \text {. }
$$

Здесь уровень замеров температур принят за нуль, снижение температуры принято в первом варианте мгновенно равным $-\Delta T_{0}$ в первом варианте, и постепенно, по экспоненциальному закону, снижается к $-\Delta T_{0}$ во втором варианте. В обоих вариантах переходим к изображениям по Лапласу, согласно формуле

$$
\Theta(x, s)=\int_{0}^{\infty} e^{-s t} T(x, t) d s .
$$

Получаем для изображений обыкновенное дифференциальное уравнение 2-го порядка с условиями в нуле и бесконечности 


$$
\left.\left.s \Theta=a \Theta^{\prime \prime}, \Theta(\infty, s)=0,1\right) \Theta(0, s)=-\frac{\Delta T_{0}}{s}, 2\right) \Theta(0, s)=-\frac{\Delta T_{0} \alpha}{s(s+\alpha)} .
$$

Оба решения имеют в изображениях достаточно простые представления

$$
\text { 1) } \left.\Theta(x, s)=-\frac{\Delta T_{0}}{s} e^{-\sqrt{\frac{s}{a}} x}, \quad 2\right) \Theta(x, s)=-\frac{\Delta T_{0} \alpha}{s(s+\alpha)} e^{-\sqrt{\frac{s}{a}} x} .
$$

Однако во втором варианте при обращении возникают трудности. Ограничимся только определением потока тепла с единицы поверхности в том и другом случаях. Обозначив через $\lambda$ теплопроводность породы, для потока тепла в изображениях при $x=0$, дифференцированием (1.1), имеем

$$
\text { 1) } \left.-\lambda \Theta^{\prime}(0, s)=-\frac{\lambda \Delta T_{0}}{\sqrt{a s}}, \quad 2\right)-\lambda \Theta^{\prime}(0, s)=-\frac{\lambda \Delta T_{0} \alpha}{\sqrt{a s}(s+\alpha)} .
$$

Несмотря на незначительность изменения граничного условия, пакет Mathcad отказывает в символьном обращении второго варианта изображения (1.2). Дело в том, что второе решение содержит полюс на отрицательной полуоси комплексной плоскости $s$ и требуется детализация контура интегрирования.

Согласно формуле Меллина оригинал от изображения $F(s)$ можно представить контурным интегралом (если $F(s) \rightarrow 0$ для $\operatorname{Re}(s)<0$ и при $s \rightarrow \infty$ ) (см. [1, 3])

$$
f(t)=\frac{1}{2 \pi i} \int_{\sigma-i \infty}^{\sigma+i \infty} e^{s t} F(s) d s
$$

Здесь контур представляет собой прямую линию $\operatorname{Re}(s)=\sigma$, где левее $\sigma>0$ расположены все особые точки изображения, а в области аналитичности контур можно деформировать. В нашем случае нулевая точка есть точка ветвления, наличие квадратного корня обязывает ввести разрез вдоль отрицательной полуоси $\operatorname{Re}(s)<0$. Контур интегрирования, находясь в области аналитичности $(1.2)$, заменяем составной линией: от $-\infty$ вдоль нижнего берега разреза до точки на оси $s=-\alpha-\varepsilon$; далее по полуокружности радиуса $\varepsilon$ с центром в точке $s=-\alpha$; нижний берег от $s=-\alpha+\varepsilon$ до нуля; верхний берег отрицательной полуоси до $s=-\alpha+\varepsilon$; обход сверху полюса по полуокружности; по верхнему берегу отрицательной полуоси до $-\infty$. Вариантам (1.2) нашей задачи соответствуют потоки количества теплоты на единицу площади, определяемые по формулам

$$
\text { 1) } \left.-\frac{\lambda \Delta T_{0}}{\sqrt{a s}} \rightarrow-\frac{\lambda \Delta T_{0}}{\sqrt{\pi a t}}, \quad 2\right)-\frac{\lambda \Delta T_{0} \alpha}{(s+\alpha) \sqrt{a s}} \rightarrow-\frac{2 \lambda \Delta T_{0} \alpha \sqrt{t}}{\pi \sqrt{a}} \int_{0}^{\infty} \frac{e^{-\eta^{2}}}{\alpha t-\eta^{2}} d \eta \text {. }
$$

При интегрировании вокруг полюса во второй задаче получается нуль. Значение несобственного интеграла следует понимать в главном смысле. Пакет Mathcad справляется с такого рода квадратурами, если расписать интеграл в виде суммы с выделением особенностей. 
Первая формула показывает, что извлекаемая тепловая энергия во времени убывает обратно пропорционально квадратному корню. Для восстановления извлекаемого теплового потока со временем придётся искать всё новые и новые источники добычи мощностей. Отношение второго потока к первому записываем в виде суммы с выделением особенностей, вводя достаточно малое $\varepsilon$

$$
q(\alpha t)=\frac{2 \alpha t}{\sqrt{\pi}}\left\{\int_{\varepsilon}^{\sqrt{\alpha t}-\varepsilon} \frac{e^{-\eta^{2}}}{\alpha t-\eta^{2}} d \eta+\int_{\sqrt{\alpha t}+\varepsilon}^{10} \frac{e^{-\eta^{2}}}{\alpha t-\eta^{2}} d \eta\right\} .
$$

На рис. 1 дано изображение отношений потоков как функции от $\alpha t$, которое представляет собой безразмерную переменную.

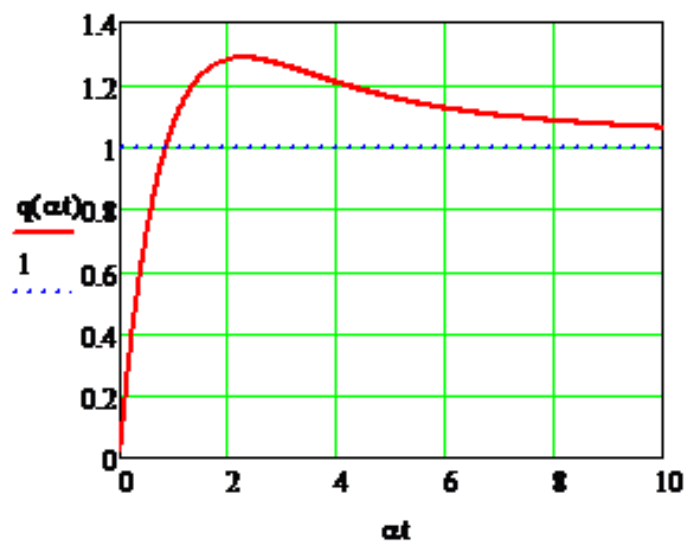

Рис. 1. Отношение потока тепла с постепенным снижением температуры к потоку тепла с мгновенным его снижением на поверхности полуплоскости.

\section{2. Постановка задачи}

Рассмотрим нестационарную задачу фонтанирования воды вверх по вертикальной трещине или разлому с постоянной скоростью. Источником воды служит глубоко расположенный пласт, где температура значительно превосходит температуру на поверхности. Считаем также, что пластовое давление обеспечивает стабильное фонтанирование по естественной трещине. В начальный момент распределение температуры по накрывающим пласт горным породам имеет линейное распределение, которое соответствует геотермическому градиенту и убывает от пластовой температуры до поверхностного значения. Для общности и последующего применения к скважинам будем полагать, что трещина может быть обсажена и металлическим каркасом.

Выберем начало координат $z=0$ на уровне пласта, ось $O z$ направим вертикально вверх по течению, ось $O x$ направим горизонтально вдоль пласта вправо 
от поверхности щели или точки контакта каркаса с горной породой. Введём следующие обозначения: $2 h$ - раскрытость трещины, в м; $\delta$ - толщина каркаса; $\Gamma$ - геотермический градиент, $\Gamma \approx 0.03^{\circ} \mathrm{C} / \mathrm{M} ; c_{\mathrm{B}}, c_{\mathrm{M}}$ и $c$ - объёмные удельные теплоемкости добываемой воды, металлического каркаса и горной породы; $T_{\text {пл }}$ - пластовая температура, равная температуре жидкости при $z=0 ; T_{\Gamma}(z)$ - температура горной породы вдали от трещины, $T_{\Gamma}(z)=T_{\text {пл }}-\Gamma z ; T_{\mathrm{B}}(z, t)-$ усреднённая температура воды и металла в трещине на данном уровне $z$. Вводим и усреднённое значение объёмной удельной теплоёмкости обсаженной щели как средневзвешенного по объёму воды и каркаса (при отсутствии каркаса $\delta=0$ )

$$
c_{k}=\frac{c_{\mathrm{B}} h+c_{\mathrm{M}} \delta}{h+\delta}
$$

Начальное распределение температуры вертикальной трещины с водой естественно принять тоже линейным, $T_{\mathrm{B}}(z, 0)=T_{\text {пл }}-\Gamma z$ с геотермальным градиентом Г, как и температуру горной породы вдали от скважины. Со временем в трещине температура воды возрастает, этот рост передается каркасу и горной породе. Под $T_{\mathrm{B}}(z, t)$ понимается усреднённое (вместе с каркасом, если он есть) по поперечному сечению трещины значение. Основанием такого усреднения служит высокая теплопроводность металла, на порядок и более превосходящая теплопроводность горной породы или воды.

При этих предположениях нестационарная задача роста температуры в трещине описывается двумя дифференциальными уравнениями (для горной породы и для самой щели), начальными условиями, условием ограниченности на бесконечности решений, а также контактным условием на границе между трещиной и горной породой.

$$
\begin{gathered}
\frac{\partial T}{\partial t}=a \frac{\partial^{2} T}{\partial x^{2}}, \quad a=\frac{\lambda}{c}, \quad x>0, \quad t>0 \\
T(x, z, 0)=T(\infty, z, t)=T_{\text {пл }}-\Gamma z ; \quad T(0, z, t)=T_{k}(z, t) . \\
c_{k} \cdot 2 h \frac{\partial T_{k}}{\partial t}+c_{\mathrm{B}} Q_{\mathrm{B}} \frac{\partial T_{k}}{\partial z}=2 \lambda T_{x}^{\prime}(0, z, t), \\
T_{k}(0, t)=T_{\text {пл }} ; \quad T_{k}(z, 0)=T_{\text {пл }}-\Gamma z, \quad z>0 .
\end{gathered}
$$

Основная цель - профили температуры колонны во времени и потери тепла извлекаемой воды из-за оттока в горную породу. Вводить глубину залегания пласта до решения задачи нет смысла, переменная $z$ определит её значение, а в формулировке задачи становится одним геометрическим размером меньше. Высоту горньх пород можно полагать бесконечной, а характерный линейный параметр вертикали определится естественным образом в процессе перехода к безразмерным переменным.

\section{3. Приведение к безразмерному виду}

Вводим безразмерную разность температур воды и горной породы вдали от трещины, отнесённую к геотермическому перепаду на характерной длине $l$ 


$$
\begin{gathered}
\xi=\frac{r}{(h+\delta)}, \quad \zeta=\frac{z}{l}, \quad \tau=\frac{a t}{(h+\delta)^{2}}, \quad l=\frac{c c_{\mathrm{B}} Q_{\mathrm{B}}}{\pi \lambda c_{\mathrm{K}}} . \\
\vartheta(\xi, \zeta, \tau)=\frac{1}{\Gamma l} \Delta T(\xi, \zeta, \tau), \quad \vartheta_{\mathrm{\kappa}}(\zeta, \tau)=\frac{1}{\Gamma l} \Delta T_{\mathrm{K}}(\zeta, \tau), \\
\Delta T(r, z, t)=T(r, z, t)-T_{\text {пл }}+\Gamma z, \quad \Delta T_{\mathrm{\kappa}}(z, t)=T_{\mathrm{K}}(z, t)-T_{\text {пл }}+\Gamma z, \\
\Theta(\xi, \zeta, s)=\int_{0}^{\infty} e^{-s \tau} \vartheta(\xi, \zeta, \tau) d \tau, \quad \Theta_{\mathrm{\kappa}}(\zeta, s)=\int_{0}^{\infty} e^{-s \tau} \vartheta_{\mathrm{K}}(\zeta, \tau) d \tau .
\end{gathered}
$$

С ростом времени температура в трещине растёт до значения пластовой температуры, потому распределение $\Delta T_{\mathrm{K}}(z, t) \rightarrow \Gamma z$, тогда как вдали от трещины для горной породы при $x \rightarrow \infty$ будет $\Delta T \rightarrow 0$. В безразмерных обозначениях исходная задача для горной породы принимает универсальный вид, все размерные параметры задачи прячутся в безразмерные переменные (3.1)

$$
\begin{gathered}
\frac{\partial \vartheta}{\partial \tau}=\frac{\partial^{2} \vartheta}{\partial \xi^{2}}, \quad \xi>0, \quad \tau>0, \quad \zeta>0 \\
\vartheta(\xi, \zeta, 0)=\vartheta(\infty, \zeta, \tau)=0 ; \quad \vartheta(0, \zeta, \tau)=\vartheta_{\mathrm{K}}(\zeta, \tau), \\
\frac{\partial \vartheta_{k}}{\partial \tau}+\frac{\partial \vartheta_{k}}{\partial \zeta}=1+\vartheta_{\xi}^{\prime}(0, \zeta, \tau), \quad \vartheta_{k}(0, \tau)=0, \vartheta_{k}(\zeta, 0)=0 .
\end{gathered}
$$

\section{4. Решение задачи}

Для решения задачи воспользуемся операционным методом Лапласа. Переходя к изображениям, получаем 2 зацепленные краевые задачи для обыкновенных дифференциальных уравнений с условиями ограниченности на бесконечности и контактным условием при $\xi=0$

$$
\begin{array}{ll}
s \Theta=\frac{d^{2} \Theta}{d \xi^{2}}, \quad \Theta(\infty, \zeta, s)<\infty, & \Theta(0, \zeta, s)=\Theta_{k}(\zeta, s), \\
s \Theta_{k}+\frac{d \Theta_{k}}{d \zeta}=\frac{1}{s}+\Theta_{\xi}^{\prime}(0, \zeta, s), & \Theta_{k}(\zeta, s)<\infty, \quad \Theta_{k}(0, s)=0 .
\end{array}
$$

Решение первой краевой задачи определяется убывающей экспонентой, откуда находим значение производной на границе породы и трещины

$$
\Theta(\xi, \zeta, s)=\Theta_{k}(\zeta, s) e^{-\sqrt{s} \xi}, \quad \Theta_{\xi}^{\prime}(0, \zeta, s)=-\Theta_{k}(\zeta, s) \sqrt{s} .
$$

Подставив найденное значение производной в (4.1), имеем задачу

$$
\frac{d \Theta_{k}}{d \zeta}+(s+\sqrt{s}) \Theta_{k}=\frac{1}{s}, \quad \Theta_{k}(0, s)=0 .
$$

Решение для изображения распределения температуры вверх по трещине представимо в достаточно простом виде

$$
\Theta_{k}(\zeta, s)=\frac{1-e^{-(s+\sqrt{s}) \zeta}}{s(s+\sqrt{s})} .
$$




\section{5. Обращение}

Применение формулы Меллина для обращения оказалось неприемлемым непосредственно в виде (4.2). Виной тому наличие температурных волн в оригинале, они увеличивают погрешности при контурном интегрировании. Множитель экспоненты с показателем $-s \zeta$ означает, что оригинал содержит волну от разности $\tau-\zeta$, равную 0 при $\tau<\zeta$. Эту волну желательно выделить, погрешность снижается. Изображение (4.2) с выделенной волной

$$
\Theta_{\mathrm{K}}(\zeta, s)=\frac{1}{s(s+\sqrt{s})}-e^{-s \zeta} \frac{e^{-\zeta \sqrt{s}}}{s(s+\sqrt{s})}
$$

следует обращать 2 раза, сначала брать обращение первой дроби, оно и есть оригинал до прихода волны в точку $\zeta$, а затем вычитается оригинал второй дроби с заменой временного аргумента $\tau$ на разность $\tau-\zeta$. Пользуясь таблицами [3; стр. 211 и стр. 221], находим обращения дробей, более входящих в $(5.1)$

$$
\begin{aligned}
& \frac{b^{2}}{s(s+b \sqrt{s})} \div \frac{2}{\sqrt{\pi}} b \sqrt{\tau}+e^{b^{2} \tau} \operatorname{erfc}(b \sqrt{\tau})-1, \quad \operatorname{erfc}(x)=\frac{2}{\sqrt{\pi}} \int_{x}^{\infty} e^{-x^{2}} d x, \\
& \frac{b^{2} e^{-b \zeta \sqrt{s}}}{s(s+b \sqrt{s})} \div\left\{e^{b^{2}(\zeta+\tau)} \operatorname{erfc}\left(b \sqrt{\tau}+\frac{b \zeta}{2 \sqrt{\tau}}\right)-\left(1+b^{2} \zeta\right) \operatorname{erfc}\left(\frac{b \zeta}{2 \sqrt{\tau}}\right)+\frac{2 b \sqrt{\tau}}{\sqrt{\pi}} e^{-\frac{b^{2} \zeta^{2}}{4 \tau}}\right\} .
\end{aligned}
$$

В отличие от изображения (5.2), во втором слагаемом асимптотического решения (5.1) содержится множитель $\exp (-\zeta s)$, который приводит к наличию бегущей волны $[6,7]$ в оригинале, согласно соответствию

$$
F(s) \div f(\tau), \quad \exp (-\zeta s) F(s) \div f(\tau-\zeta),
$$

причем значения оригинала $f(\tau-\zeta)$ равны нулю при отрицательных значениях его аргумента, т.е. если $\tau<\zeta$. Это значит, что оригинал от второго слагаемого в формуле (5.1) присутствует лишь в той области $\tau>\zeta$, куда уже пришёл тепловой фронт от забоя, и отсутствует в области $\tau<\zeta$, куда еще тепловой фронт не дошёл. При обращении второго слагаемого (5.1) переменную $\tau$ заменяем разностью $\tau-\zeta$.

Чтобы упростить запись оригинала, введем в рассмотрение еще две функции, через которые выражаются оригиналы (5.1) в безразмерных переменных

$$
\begin{gathered}
\theta_{1}(t)=e^{t} \operatorname{erfc}(\sqrt{t})-1+\frac{2}{\sqrt{\pi}} \sqrt{t} \\
\theta_{2}(z, t)=e^{t+z} \operatorname{erfc}\left(\sqrt{t}+\frac{z}{2 \sqrt{t}}\right)-(1+z) \operatorname{erfc} \frac{z}{2 \sqrt{t}}+\frac{2 \sqrt{t}}{\sqrt{\pi}} e^{-\frac{z^{2}}{4 t}} .
\end{gathered}
$$

Для распределения температуры вдоль трещины (оригинала) получаем 


$$
\vartheta_{:}(\zeta, \tau)=\mid \begin{aligned}
& \theta_{1}(\tau), \quad 5 A ; 8 \quad \tau \leqslant \zeta \\
& {\left[\theta_{1}(\tau)-\theta_{2}(\zeta, \tau-\zeta)\right], \quad 5 A ; 8 \tau>\zeta .}
\end{aligned}
$$

На рис. 2 показано поведение решения (5.5) во времени как семейства функций для указанных уровней высот $\zeta$. С ростом времени $\tau$ темпы прироста температуры резко ослабевают.

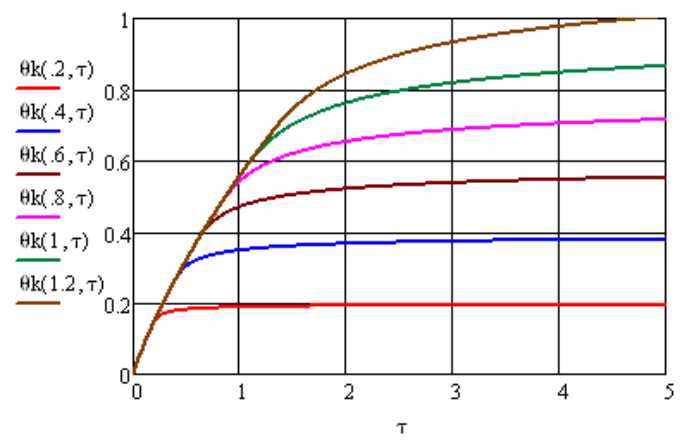

Рис. 2. Прирост температуры по трещине во времени для различных уровней $\zeta$.

На рис. 3 показаны профили прироста температуры вдоль колонны скважины, для некоторых фиксированных значений $\tau$. По ним видно, что с некоторого уровня прирост температуры для всех $\zeta$ становится одинаковым, видно наличие фронта температур.

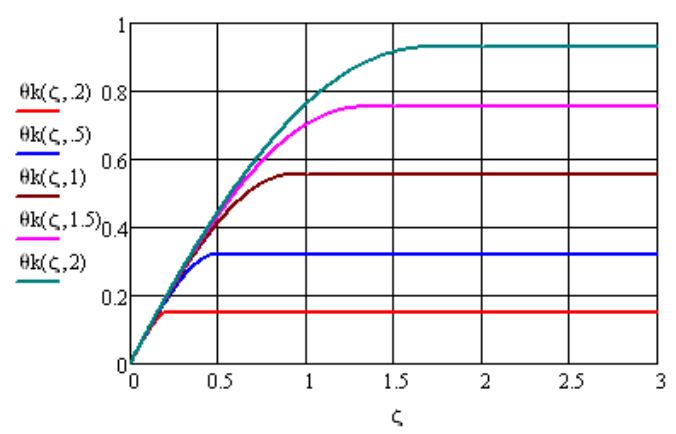

Рис. 3. Профили прироста температуры жидкости по трещине для некоторых $\tau$.

Профили безразмерной температуры на рис. 2 и рис. 3 соответствуют небольшим значениям времён. На рис. 4 представлена поверхность температур в логарифмическом масштабе времени.

Формулы (5.3), (5.4) и (5.5) хороши тем, что дают точное решение задачи в элементарных функциях. Однако считать по ним можно для относительно небольших времён. В нефтяном деле и геотермальных проблемах расчётные 


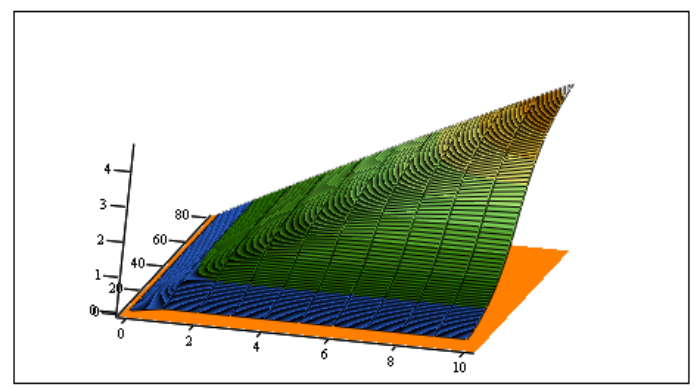

$\Delta$

Рис. 4. Поверхность прироста температур в логарифмическом масштабе времени. По лицевой оси отложены значения $\zeta_{n}=0.5 n, n=10$ соответствует $\zeta=5$. По второй горизонтальной оси отложены значения $\tau_{k}=0.1 \cdot 1.1^{k}, k=80$ соответствует $\tau=60$ (примерно 9 суток).

оценки приходится получать для десятков лет, а в представленном виде эти формулы для больших времён не пригодны. Дело в том, что содержащаяся в них экспоненциальная функция стремительно растёт, а множитель рядом убывает к нулю. Для больших времён необходим переход на асимптотические представления с оценкой погрешности. Приводим некоторые пояснения, прежде чем считать для больших времён.

Приведём некоторые промысловые данные для нашей задачи прогноза роста температуры добываемой воды. На Тарумовском месторождении в Дагестане забои скважин вскрыты на глубинах $H \approx 5400$ м. Значения теплофизических параметров принимались такими: для горной породы $\lambda=2.5 \mathrm{~B} \mathrm{~T} /\left(\mathrm{м} \cdot{ }^{\circ} C\right)$, $c=3.2$; для термальной воды $c_{\mathrm{B}}=4.19$; для металла труб $c_{\mathrm{M}}=3.46$; для эксплуатационной колонны $\left.c_{\mathrm{K}}=3.82 \mathrm{MДж/} / \mathrm{m}^{3} \cdot{ }^{\circ} C\right)$. Среднегодовая температура нейтрального слоя $T_{0}=15^{\circ} \mathrm{C}$, температура пластовой жидкости на забое предположительно $T_{\text {пл }}=195^{\circ} \mathrm{C}$, расстояние от забоя до устья $5400 \mathrm{м}$, геотермический градиент $1^{\circ} \mathrm{C}$ на 30 м. Максимально прирост температуры на нейтральном слое может составить до $180^{\circ} \mathrm{C}$. Дебиты скважин на Тарумовке значительно разнятся друг от друга, но имеют достаточно высокие значения: от нескольких сотен м ${ }^{3}$ в сутки и до 10 тысяч. Температура на устье с течением времени нарастает до значения, которое меньше пластового. Замеренное на устье значение температуры составило $171^{\circ} \mathrm{C}$ при дебите $Q_{\text {в }} \approx 6800 \mathrm{~m}^{3}$ в сутки, что ниже пластовой температуры на $24^{\circ} \mathrm{C}$. Темпы нарастания зависят от дебита, почти пропорциональны дебиту.

Нашумевшее после 200-летней спячки извержение вулкана Исландии Эйяфьятлайокудль («Остров горных ледников») началось 21 марта 2010 года выбросом горячих газов по трещине длиной 500 м и существенно усилилось с 14 апреля 2010 года выбросами газов, пепла и лавы из 3-х жерл диаметрами около 200, 150 и 100 м. Температура выбрасываемых масс достигала до $1300^{\circ} \mathrm{C}$ и более, что свидетельствовало их подъём с глубин более 40 км. Первые три дня вулкан выбрасывал вместе с газами 600 - 800 тонн пепла в секунду. На 20 апреля над вулканом был виден темно-серый столб пепла высотой 8.5 км. 
Эти данные довольно скудны, ограничиваемся лишь приведением ориентировочных расчётов. Полагаем $\delta=0, h=0.1$ м, ширина трещины $2 h=20$ см. Геологами замечены разломы до 50 см раскрытости. Глубину залегания продуктивного горизонта примем 5400 м, а геотермический градиент равным $\Gamma=1 / 30{ }^{\circ} \mathrm{C} / \mathrm{m}$. Теплофизические параметры воды и горных пород примем такими же, как для Тарумовки. Тогда характерное время $\tau=1$ составит 0.148 суток при температуропроводности около $0.0675 \mathrm{~m}^{2} /$ сутки, причём изменение дебита или средней скорости течения по трещине не влияет на характерное время задачи. При выбросах воды на погонный метр $Q_{\text {в }}=425$ кубов в сутки средняя скорость по сечению трещины составит $25 \mathrm{mм} / \mathrm{c}$, а характерный масштаб $\zeta=1$ соответствует $l=500$ м. Увеличение объёма выбросов (или средней скорости течения) ведёт к пропорциональному росту масштаба $l$. Гра-

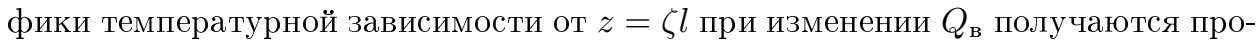
порциональным растяжением-сжатием вертикальной оси одного сосчитанного варианта.

Особенность тепловых расчётов для задач нефтепромысловой механики в том, что прогнозные характеристики выдаются на большие времена, на 30 50 лет и более. Время 30 - 50 лет соответствует безразмерным $\tau$ от 75000 до 125000 , а для таких значений $\tau$ ни один пакет программ не будет считать экспоненциальные функции, входящие в формулы (5.3) и (5.4). Точное решение найдено в простом виде (5.5), но считать по нему в пакете Mathcad можно только тогда, когда $\tau$ небольшое, примерно для $\tau<500$. Для $\tau>500$ формулы (5.3) и (5.4) следует заменить, исключив из первых их слагаемых употребление экспоненты, используя наличие такой же экспоненты с противоположным знаком в асимптотическом поведении функции $\operatorname{erfc}(x)$. Обращение к асимптотическому поведению решения для $\tau>500$ становится необходимостью.

Согласно [7], для больших значений аргумента $z$ имеет место асимптотика

$$
\sqrt{\pi} z e^{z^{2}} \cdot \operatorname{erfc}(z) \approx 1+\sum_{m=1}^{\infty}(-1)^{m} \frac{1 \cdot 3 \ldots(2 m-1)}{\left(2 z^{2}\right)^{m}} .
$$

Она позволяет заменить функции $\theta_{1}(t)$ и $\theta_{2}(z, t)$ другими формулами с оценкой погрешности, где экспоненты заменены разложениями по отрицательным степеням временной переменной. Например, первую функцию $\theta_{1}(t)$ удобнее считать с использованием оператора или функции if в двойном виде $\left(t_{0}=500\right)$

$$
\begin{gathered}
\theta_{1}(t)=i f\left(t<t_{0}, \frac{2 \sqrt{t}}{\sqrt{\pi}}-1+e^{t} \cdot \operatorname{erfc}(\sqrt{t}),\right. \\
\left.\frac{2 \sqrt{t}}{\sqrt{\pi}}-1+\frac{1}{\sqrt{\pi t}}-\frac{1}{2 t \sqrt{\pi t}}+\frac{1 \cdot 3}{4 t^{2} \sqrt{\pi t}}-\ldots\right) .
\end{gathered}
$$

Погрешность асимптотики определяется значением отброшенного члена ряда. При $t_{0}=500$ значение последнего написанного члена ряда равно $0.75 \cdot 10^{-7}$, его даже можно упустить. Если же принять $t_{0}=100$, то его значение $0.42 \cdot 10^{-5}$. На рис. 5 приведён график функции $\theta_{1}$ в логарифмическом масштабе времени, сосчитанный для больших времён с использованием асимптотики, а точками отмечены возможный интервал счёта по формуле (5.3). Принято для $\theta a(\tau 0)=100$. 


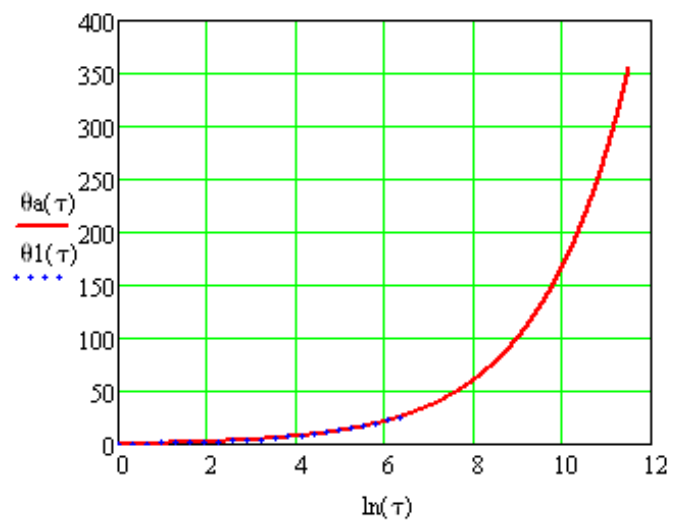

Рис. 5. Сравнение расчётов по формулам (5.6) и (5.3) функции $\theta_{1}(\tau)$ для случая использования асимптотического разложения $\theta a$ и без такового использования $\theta 1$. Интервалы счёта для обеих функций задавались одинаково. По формуле (5.3) пакет не считает при $\tau>544$.

Аналогично (5.6) и функцию $\theta_{2}(z, t)$ можно представить в считаемом виде

$$
\begin{gathered}
\theta_{2}(z, t)= \\
i f\left(\begin{array}{l}
t<t_{0}, \frac{2 \sqrt{t}}{\sqrt{\pi}} e^{-\frac{z^{2}}{4 t}}-(1+z) \operatorname{erfc} \frac{z}{2 \sqrt{t}}+e^{t+z} \operatorname{erfc}\left(\sqrt{t}+\frac{2}{2 \sqrt{t}}\right), \\
\frac{e^{-\frac{z^{2}}{4 t}}}{\sqrt{\pi}}\left[2 \sqrt{t}+\frac{1}{\sqrt{t}+\frac{z}{2 \sqrt{t}}}-\frac{1}{2\left(\sqrt{t}+\frac{z}{2 \sqrt{t}}\right)^{3}}+\frac{1 \cdot 3}{4\left(\sqrt{t}+\frac{z}{2 \sqrt{t}}\right)^{5}}\right]-(1+z) \operatorname{erfc} \frac{z}{2 \sqrt{t}}
\end{array}\right) .
\end{gathered}
$$

При очень больших временах можно упростить ещё асимптотику, с рядом оговорок, но и представленный вид довольно хорошо справляется со счётом. На рис. 6 представлен график прироста размерных значений температуры на устье скважины глубиной 5400 метров для условий Тарумовки, $\Delta T U_{j}(11, \tau)$ дано в логарифмическом масштабе безразмерного времени. Счёт производился по равным промежуткам логарифма для 130 точек. В размерном виде интервал времени соответствует 36 годам. Из графика видно, что выход на прямую линию не наблюдается. Максимальный прирост $180^{\circ} \mathrm{C}$ достигается довольно поздно.

\section{Заключение}

Предложена модель для задачи определения роста температуры добываемой термальной воды в строго нестационарной постановке с учётом обмена тепла с горными породами. Для случая подъёма вод по трещине она сводится к 


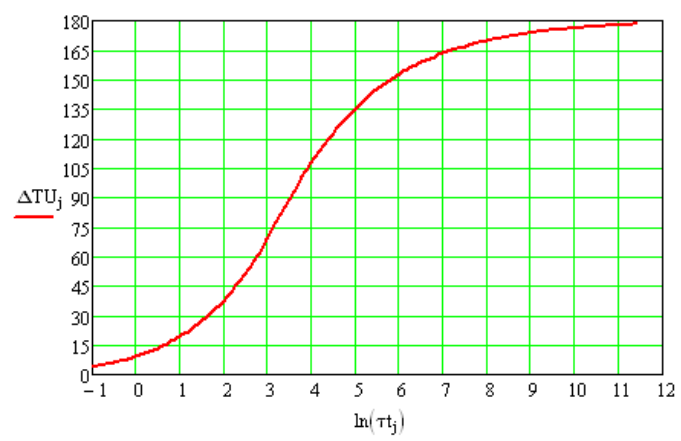

Рис. 6. Изображение размерного прироста температуры в логарифмической шкале для условий Тарумовского месторождения термальных вод.

универсальному виду подбором безразмерных переменных и имеет точное аналитическое решение, поддающееся расчёту достаточно простыми средствами.

\section{Список литературы}

[1] Деч Г. Руководство к практическому применению преобразования Лапласа. М.: Наука. 1965. 287 с.

[2] Чекалюк Э.Б. Термодинамика нефтяного пласта. М.: Недра. 1965. 238 с.

[3] Бейтмен Г., Эрдейи А. Таблицы интегральных преобразований. Преобразование Фурье, Лапласа, Меллина. М.: Наука. 1968. 344 с.

[4] Тихонов А.Н., Самарский А.А. Уравнения математической физики. М.: Наука. 1972. $735 \mathrm{c.}$

[5] Пехович А.И., Жидких В.М. Расчёты теплового режима твердых тел. Л.: Энергия. 1976. $351 \mathrm{c.}$

[6] Алишаев М.Г. Уточнение потерь тепла для геотермальной скважины // Известия РАН. Энергетика. 2010. № 1. С. 36-47.

[7] Справочник по специальным функциям с формулами, графиками и математическими таблицами. Под редакцией М. Абрамовица и И. Стиган. М.: Наука. 1972. $832 \mathrm{c}$.

М. Г. Алишаев (M. G. Alishaev)

Поступила в редакцию

Дагестанский научный центр РАН,

08.12 .2015

Институт проблем геотермии Дагестанского научного центра РАH

E-mail: alishaev@rambler.ru 